\title{
LO COTIDIANO (ENTRE USOS Y RESISTENCIAS) DE LAS TIC EN UN AULA DE LA EDUCACIÓN SECUNDARIA OBLIGATORIA. Apuntes etnográficos
}

\author{
José Antonio CAMPOS MARTÍNEZ \\ Universidad de Granada (España) \\ jacamposmartinez@yahoo.es
}

\section{EVERYDAY USES AND RELUCTANCES ICTS IN A SECUNDARY EDUCATION CLASSROOM. Etnographic notes}

Resumen: Existe una tendencia a considerar las TIC como una amenaza y ello a pesar del creciente uso en integración en la sociedad y, en especial, en los sistemas educativos. La inclusión de estas en la escuela ofrece una gran cantidad de resistencias que tienen que ver con todos los agentes participantes. Padres y madres, alumnado y profesorado juegan un papel fundamental en dicha resistencias, a la vez que son estos los que tienen que propiciar su integración. Pero para poder comprender mejor estos procesos de resistencias y usos de las TIC resulta imprescindible la inmersión en el contexto en el que confluyen las tensiones referidas. Lo que se pretende con este artículo es poner en dialogo los usos de las TIC y las resistencias que se observan en comportamientos y que se escuchan en discursos en un contexto escolar de una ciudad andaluza. Mediante un acercamiento etnográfico a un centro educativo privado concertado y al conjunto de su comunidad educativa, pretendemos describir las barreras con las que se etiquetan las TIC en las aulas y las costumbres que se han instalado en las mismas.

Abstract: There is an extended opinion to consider ICT a threat in spite of its increasing use and full integration in society, especially in education systems. The inclusion of ICT in school curriculum shows a considerable reluctance among those who take an active part in education systems. Parents, students and teachers play a key role in such reluctance, although they are the ones who should promote their integration. However, for a better understanding of ICT in school, it is essential the immersion in the context in which the aforementioned tensions converge. The aim of this article is to set up a debate of the use of ICT and the negative attitudes observed and heard in a school context of an Andalusian city towards ICT. From an ethnographic point of view, we intend to describe the prejudices that label ICT a negative thing to be used in classrooms and the customs assumed in a state-subsidised school and its educational community.

Palabras clave: TIC. Etnografía. Escuela. Innovación educativa. Andalucía ICT. Ethnography. School. Educational Innovation 


\section{Acercamiento a un contexto escolar. El hacer de un maestro etnógrafo}

Aunque el éxito del aprendizaje no sea inherente a la inclusión de las nuevas tecnologías en educación, "dejar de lado el uso de las TIC es privar a la educación de posibilidades insospechadas y dejar de lado una excelente oportunidad para que los maestros innoven" (Vásquez Rodríguez, 2015). Es decir, resulta significativo que la sociedad deje de lado una realidad que integra a toda la sociedad de una forma global. Pero las resistencias son cada vez menores y la adaptación de la sociedad a este compromiso, progresivo. En este sentido, adquieren especial relevancia para este trabajo aspectos como el cariz del adolescente nativo digital, el ensimismamiento por la ubicuidad (De Pablos, 2015), el predominio de la redes sociales para comunicarse (Âlvarez de Sotomayor, 2011), la importancia de la construcción de la identidad mediada por la aparición de las nuevas tecnologías (Francecs, 2015), la importancia de superación del tópico de la brecha digital (Garrote Pérez de Albéniz, 2013) y la inherente capacidad del ser humano por integrar los avances tecnológicos en su Realidad. También es importante contemplar las implicaciones éticas de la tecnología misma y de sus uso, pues esta reflexión no puede aislarse del resto de aspectos (Aguado, 2003).

Al final, las TIC no hacen sino hablarnos de la innovación educativa (del cambio en la educación). Y en este sentido debemos recordar que "no ha habido innovación tecnológica u organizativa en la educación que no trajera consigo una amplia discusión sobre el presente o el futuro del profesorado" (Fernández Enguita, 2013: 145), discusión que sigue presente de forma exponencial ante el inevitable atractivo digital.

En este contexto de innovación educativa es incuestionable que el profesorado juega un papel fundamental y para estudiar dicho papel se hace imprescindible acercarse a dicho profesorado, pero no solo a él. Este profesorado representa su identidad a la hora de transmitir sus inquietudes y novedades en el proceso de enseñanza-aprendizaje como un constructo, formado a la vez por factores racionales y no racionales, integrado en el contexto de un centro educativo con familias y alumnado. Los centros educativos son agentes de modernización en los que los educadores pueden hacer de etnógrafos de la escuela para investigar en un contexto de instrumentalización de las relaciones sociales como único modo de acceder directamente al comportamiento social y así comprenderlo más en profundidad (Velasco Maillo, García Castaño, \& Díaz de Rada, 2007). Así, la mejor estrategia para el análisis de los grupos humanos es establecer y operacionalizar con las personas que la integran (Velasco \& Díaz de Rada, 2006) en un contexto de familiarización: "los antropólogos no estudian aldeas (tribus, ciudades, vecindarios, etc.), sino que estudian en aldeas" (Geertz, 1973: 22, citado en Velasco \& Díaz de Rada, 2006, 48).

El objetivo de esta trabajo ${ }^{1}$ ha sido describir el uso que hacen de las TIC un grupo de alumnado de un aula de un centro educativo de titularidad privada-concertada en una capital de provincia de Andalucía. El contexto ha sido el escolar, pero junto a él se han establecidos relaciones sociales con padres, madres y profesorado del centro. La perspectiva no ha consistido en centrarse exclusivamente en qué tipo de TIC utilizan, sino y muy especialmente cómo las utilizan. De manera muy concreta lo que ahora presentaremos se concreta en los siguientes puntos:

- Describir lo que piensan sobre la introducción de la tecnología en el ámbito escolar alumnos/as padres y madres, y profesorado.

- Reconocer el uso destinado a los dispositivos tecnológicos aplicados en el

$1 \mathrm{El}$ presente texto forma parte de mi tesis doctoral realizada en el Departamento de Antropología Social de la Universidad de Granada bajo la dirección de F. Javier García Castaño. En ella se desarrolla mucho más ampliamente los procesos de usos de las TIC en un centro escolar y se describen los discursos de resistencia y, en general, los comportamiento culturales asociados a dichas nuevas tecnologías. 
ámbito educativo y el espacio insalvable de la brecha digital.

- Comprender la construcción de nuevas lógicas de relaciones sociales a raíz de la aparición de las TIC.

Análisis y codificación del uso que los/las alumnos/as de $4^{\circ}$ de ESO hacen de la aplicación móvil WhatsApp, del móvil y del iPad durante el período escolar 2014-2015.

- Analizar el discurso y el contexto desde el que se generan los tópicos generativos sobre las redes sociales y las TIC.

A partir de estos objetivos pretendemos mostrar cómo usos (en defensa) y resistencias de todo este nuevo aparataje tecnológico conviven en una especia de contracción que hace que no sea fácil saber qué representan las TIC en los procesos de innovación escolar. Las aportaciones científicas de este trabajo no se circunscriben explícitamente a la obviedad significativa del análisis cuantitativo de los resultados obtenidos. El trabajo se enmarca en el ámbito de la investigación cualitativa del trabajo etnográfico propio de la antropología cultural cuyo propósito es comprender mejor las razones de interacción entre los agentes de la comunidad educativa a la hora de integrar las nuevas tecnologías a la realidad educativa. En definitiva, todo tiene que ver con el comportamiento cultural ${ }^{2}$.

Teniendo muy presente esto que decimos, debemos no olvidar que una cosa es lo que se dice y otra bien distinta lo que se hace, y para ello el análisis del discurso se hace imprescindible desde esta dimensión. La investigación cualitativa refuerza algunos aspectos de la cuantificación mediante cuestionarios a la comunidad educativa; sin embargo, el discurso generado a través de las entrevistas y los grupos de discusión matizan esta información ayudando a comprender las dificultades que generan las TIC en el contexto educativo. Todo se completa y completa con insistentes y continuadas observaciones de aquellas realidades que pretendemos entender. Como dice Ortiz, Peñaherrera, y Ortega (2012: 2), la mayoría de los estudios que se han hecho sobre educación en España, han sido preferentemente de corte cuantitativo, habiendo escasas investigaciones en profundidad de corte cualitativo sobre las repercusiones tanto organizativas como curriculares y profesionales de la introducción de las TIC en la educación. En este contexto se hace necesaria una metodología cualitativa, fundamental para comprender los procesos de interacción que se dan en este tipo de interacciones en entornos convencionales, como dicen Orellana y Sánchez (2007).

Es por ello que la base metodológica de la investigación está inspirada en la etnografía aplicada al estudio de la escuela (Velasco Maillo et al., 2007). La primera fase de la investigación se ha centrado en la aplicación de varios cuestionarios dirigidos a toda la comunidad educativa del Colegio $^{3}$, a un total de 305 alumnos y alumnas, 104 padres/madres y 38 profesores y profesoras (la composición total de centro es: alumnado 1100 y profesorado 66). Los cuestionarios se han centrado en dos aspectos relevantes: inclusión y/o exclusión del uso que se hace de los dispositivos móviles en los distintos contextos de interacción de los agentes educativos: padres y madres, profesorado y alumnado. Aunque con matices, las preguntas dirigidas a los distintos agentes de la comunidad educativa han sido las mismas, y el objetivo ha sido contrastar la visión que cada uno de ellos tienen sobre dichos aspectos. La información se ha gestionado mediante el paquete estadístico SPSS21. De todas formas,

2 No queremos dejar de apuntar que desde nuestro punto de vista no existen modelos estereotipados de cultura porque el concepto de cultura es comportamental y dinámico, y son las acciones las que actualizan los sistemas de organización de la información y no al revés (G arcía García, 1998).

3 Como ya hemos mencionado, el centro educativo estudiado es un centro privado concertado de una capital andaluza que ofrece los estudios de educación primaria y ESO bajo el concierto educativa con la administración y que también ofrece los estudios de Bachillerato. Para preservar el anonimato del centro educativo y, muy especialmente, de las personas que me han acompañado durante la investigación, utilizará la referencia de "Colegio" cada vez que hable del centro escolar y de su comunidad educativa. 
el cuestionario es una buena base para el inicio de la producción de datos, pues abarca un amplio espectro de información y a un grupo poblacional significativo. Sin embargo, aunque nos acerca a la realidad de lo social, no nos aporta detalles que ayuden a una reflexión en profundidad que nos haga conocer bien dicha realidad. Falta en muchos caos la explicación y, junto a ella, la interpretación de los significados explicados.

En una segunda fase, se han realizado entrevistas semiestructuradas a algunos agentes de la comunidad educativa, a padres y madres de alumnado de cuarto curso de la ESO y a otros miembros de la comunicad educativa que ya han pasado por el centro en cursos anteriores (disponemos de un total de 14 entrevistas). El objetivo ha sido explicar mejor los momentos de transformación del centro en su proceso de innovación educativa a través de las TIC y contrastar el grado de aceptación e implicación de los distintos agentes en dichos procesos. Para codificar los resultados se ha utilizado el programa informático NVivo. A través de él, se ha codificado la información obtenida mediante nodos y matrices de significado que han aportado resultados relevantes a los atributos clasificados.

En tercer lugar, se ha categorizado y codificado los distintos movimientos generados en tres grupos de WhatsApp de cuarto curso de la ESO durante el periodo escolar 2014-2015. Desde junio de 2014 participé de los tres grupos creados por la clase, 21 alumnos y alumnas del grupo A y 27 de cada uno de los grupos B y C, respectivamente. La codificación, también realizada con programa informático NVivo, se ha centrado en dos aspectos fundamentales: frecuencia de actividad y temática del chat, es decir, horarios de mayor actividad y participación, y si se aprecian movimientos significativos respecto del ámbito académico.

Sin embargo, uno de los aspectos más relevantes de la investigación se ha centrado en la producción de información a partir de la observación participante, propia de la investigación etnográfica, desarrollada en el centro por el investigador que, a la vez, es personal docente del $\mathrm{mismo}^{4}$.

\subsection{Algo de contexto...}

El Colegio es un centro concertado perteneciente a una orden religiosa y se encuentra enmarcado en la red de centros de titularidad católica perteneciente a la CECE (Confederación Española de Centros de Enseñanza Concertada). El centro se encuentra ubicado en el cinturón de la ciudad y fue concebido para fines sociales en una zona de expansión. Durante los años setenta se sucedieron las primeras explotaciones urbanísticas en la zona y el contexto geográfico del centro fue transformándose progresivamente, de un espacio de Vega a un entorno concurrido de vida social. Algo tuvo que ver, también, la construcción de un gran centro comercial y el paso de la autovía que circunvala la ciudad de referencia del centro. Fue un periodo de continuas adaptaciones de otros centros concertados de la zona.

\footnotetext{
4 Durante el periodo que duró la investigación impartí clases en los niveles de $3^{\circ}$ y $4^{\circ}$ de la ESO y $1^{\circ}$ de bachillerato. Aunque el marco general de este trabajo tiene como objetivo analizar a todos los agentes implicados en la educación del centro, decidí acotar la información centrándome en los grupos de $4^{\circ}$ de ESO, aquellos que generan mayores conflictos respecto a las redes sociales y el uso del teléfono móvil. Sin embargo, la parte más importante del trabajo ha sido el de la observación participante. El hecho de impartir clase en el mismo centro al que me dedico profesionalmente me han supuesto ventajas e inconvenientes. Por un lado, la ventaja de poder acceder rápidamente a la información, observación entre pasillos, gestión y control sobre las entrevistas, trámites administrativos, dedicación, etc. Cualquier momento era aprovechado para obtener información como conversaciones entre pasillos, comentarios de los alumnos durante el intercambio de clase o en actividades complementarias, desayuno con profesores, momentos de crítica tras las reuniones de claustro o después del ETCP (reuniones del equipo pedagógico), etc. El mayor hándicap ha sido el del extrañamiento, un fenómeno que me ha perseguido a lo largo de toda la investigación, fundamentalmente al principio. La realización de este trabajo de investigación en un contexto de instrumentalización de las relaciones sociales parece que es el único modo de acceder directamente al comportamiento social y así comprenderlo más en profundidad. Pero hay que realizar un ejercicio de distanciamiento para poder comprender mejor lo que está ocurriendo.
} 
Alrededor del centro hay otros colegios públicos y privados de posterior construcción.

El contexto socioeconómico del centro es de un nivel medio alto, en el que tan solo un $5 \%$ de los encuestados perciben ingresos familiares anuales inferiores a 15.000 euros, con un porcentaje de casi un $30 \%$ que perciben más de 50.000 euros. El Colegio tiene tres líneas desde 3 años de Educación Infantil hasta $4^{\circ}$ de ESO. Todos estos niveles son concertados, subvencionados por la administración pública. El Bachillerato se reduce a dos líneas por curso cuya gestión es completamente privada. El alumnado que por circunstancias económicas no pueden costearse los estudios pueden optar a becas compensatorias. El $89 \%$ de las familias afirma poseer estudios superiores.

El centro ha implementado el uso del iPad en los dos cursos de bachillerato desde el curso 2013-2014 y para el curso 2015-2016 en los niveles de $5^{\circ}$ y $6^{\circ}$ de primaria y $1^{\circ}$ de la ESO, con la idea de acabar integrando el uso de este dispositivo en todo el organigrama organizativo del este, con el correspondiente adecuado equipamiento para su gestión: Wifi, Internet, control parental, gestión para la resolución de problemas técnicos, etc.

\section{Argumentos y evidencias...}

En la vida diaria del Colegio es donde se transmite el comportamiento "natural" de los agentes a través de las distintas anécdotas y momentos que se generan en el día a día. El alumnado utiliza el móvil constantemente y el profesorado, aún no reconociéndolo, hace la vista gorda, quizá porque también lo suele utilizar o porque prefiere evitar los conflictos. Es difícil ver a un profesor dejar el móvil en la taquilla. Hay quienes piensan que no se debe utilizar este en clase; otros y otras, consideran que se puede utilizar integrándolo en la vida diaria. Esta era la valoración que un compañero del centro hacía sobre este aspecto:

Lo tengo claro. Yo utilizo lo que es más fácil para todos y lo que utiliza todo el mundo. Si es el WhatsApp, pues el WhatsApp. No voy a utilizar plataformas que nadie usa. Hay que ser prácticos. Con el WhatsApp organizo los grupos y todo el mundo se mantiene informado. Todos saben lo que tienen que hacer $y$ participan activamente. A nadie le pasa nada (Marshall, Profesor) ${ }^{5}$.

Sin embargo, otros compañeros y compañeras del centro insinuaban cumplir radicalmente el ROF (Reglamento de Orden y Funcionamiento) en lo que respecta a la sustracción de cualquier uso del móvil durante la jornada escolar: "Yo veo un móvil y lo quito inmediatamente" (Dalila, Profesora). Contrasta el hecho de que en los cuestionarios enviados al profesorado, muchos de ellos insinuaban permitir el uso del teléfono móvil. Otros, sin embargo, preferían evitar los conflictos y ser tolerantes con el uso del mismo en algunos momentos de la jornada escolar.

Si se utiliza para la vida académica, ¿cómo se puede discriminar el uso lúdico del profesional si en él está todo integrado? Esta consciencia es difícil de sostener incluso para nosotros, los profesionales de la educación. Los padres y madres también quieren tener controlado el uso que se hace del móvil en casa, y fuera, aunque el control les genera grandes momentos de tensión. Así me lo relataban algunas de las familias entrevistadas sobre sus hijos de $4^{\circ}$ de la ESO:

Al estar allí tan lejos, está acostumbrado a que todo es por WhatsApp y le tengo que decir, dejad el teléfono, el teléfono ahí y hablad que estamos aquí todos como toda la vida se ha hecho. O sea, que por eso tampoco estamos muy integrados en esos temas, porque hay que relacionarse... (Madre de Rebeca,

5 Para mantener el anonimato de las personas que han colabora en nuestra investigación hemos nombrado a cada una de ellas con un nombre ficticio seguido del rol que desempeñan en el centro. 
alumna del centro).

Algunas familias hablan de la dificultad de compatibilizarlo todo lo que quieren que sus hijos o hijas hagan a lo largo del día...

Es muy difícil esto de las nuevas tecnologías, porque tú puedes educar en eso pero hay que tener la fuerza de voluntad suficiente para saber qué es lo importante. Lo más importante en este caso es, por ejemplo, el entrenamiento de baloncesto que tiene 2 ó 3 veces por semana y pierden 3 horas cada vez. $Y$, aprovechan muy bien la tarde o no lo pueden compatibilizar. Si, además, tienen que complementarlo con el WhatsApp, no pueden estudiar. Primero el entrenamiento, luego el teléfono, y estudiar ya, lo último. Y, a lo mejor, al día echa media hora o tres cuartos, y cuando llegan del entrenamiento está muy cansado y no estudia. Y al final, ¿qué hace? ¿Deja el baloncesto para tener tiempo de estudiar y del teléfono? Pues lo veo absurdo. Entonces, si recurres solamente a su sentido común, estás perdido. ¿Qué pasa? Pues que en esta casa se genera mucha tensión por culpa del teléfono. Porque resulta que tiene dos horas de estudio en el caso de Edmond, que es insuficiente en $4^{\circ}$ de eso, y que de las dos puede aprovechar, si te digo ¿una? Porque el resto del tiempo es o con el teléfono porque dice: "no, es que estoy poniendo la alarma", o "no, es que estoy viendo a ver si han enviado los ejercicios de esto o lo otro". Entonces, ¿qué hacemos? ¿Dejamos el baloncesto para que tenga tiempo de estar con el móvil? Y su sentido común, como apeles a su sentido común solamente... (Madre de Edmond, alumno del centro).

... otras se refieren a como deberíamos hacerlo en el centro educativo, quizá para facilitar su trabajo en la casa...

Es difícil controlar el tema del uso del móvil y resulta complejo controlar esto. Yo sería más estricto con el control del móvil en clase y más tajante (Padre de Diego, alumno del centro).

No faltan quienes hablan claramente de un rechazo del móvil, aunque no de otros recursos TIC:

Y yo utilizo más que este espacio, utiliza el dormitorio pero con mi portátil. Utilizo menos las nuevas tecnologías; internet, por supuesto. Soy bastante reacio al móvil (Padre de Everest, alumno del centro).

También están las personas que añoran el pasado pero parecen comprender el presente...

Y que lee, que siempre le gustó mucho leer y no está, Porque tienes el teléfono al lado y tienes un libro ¿qué haces? Te tiras a por el teléfono, es lógico, en esa edad es lógico. A ver lo que ha puesto menganita... Le puse hace una hora no sé cuánto y no me ha contestado, ah, me ha contestado entonces le pongo otra cosa. Cuanto te quiero, cuanto me dejas de querer... Yo lo entiendo, yo con esa edad haría lo mismo (Padre de Zuma, alumno del centro).

Y no faltan las sanciones morales sobre estos nuevos "cacharros":

Eso es fundamental, me acuerdo lo dijo Pedro a principio de curso, eso es 
fundamental. El teléfono es un diablo que tienes ahí que te está siempre tentando, que cuando toca un pitido te están mandando un mensaje, que a lo mejor no es para ti, que es para el grupo, pero tú te metes. En ese momento te pierdes la poca o la mucha concentración que tengas en ese tema (Padres de Edmond, alumno del centro).

A todas estas valoraciones se añaden unas nuevas prácticas de comunicación no menos interesantes. Nos referimos a la popularidad generada por compartir espacios entre profesorado y alumnado, que es cada vez mayor. Muchos alumnos y alumnas tienen el teléfono móvil de sus "profes" y los han añadido a su círculos de redes. Algunos están cambiando su actitud adentrándose en un clima de una mayor cordialidad. La interacción entre padres y madres y profesorado es cada vez mayor, fundamentalmente porque comparten espacios e intereses a través de las actividades complementarias y extraescolares que el centro fomenta (deportes, fiestas, celebraciones locales, carrera popular o el día del deporte), profesores que tienen a sus hijos en el centro y comparten reuniones, AMPA, comedor, aula matinal, y conversaciones entre pasillos). Algunos comparten también WhatsApp de clase creándose otros grupos alternativos, ampliando el contexto de relaciones, algunas de ellas pudiéndose convertir en relaciones de amistad. Más aún, antiguos alumnos y alumnas que mantienen contacto con el Colegio acaban por involucrarse en él estableciendo incluso, relaciones clientelares.

\subsection{Peligros, controles y éxitos académicos}

Uno de los síntomas sobre la llamada "brecha digital" tiene que ver con el hecho de que el $95 \%$ de los profesores tienen Internet en casa, y el $99 \%$ de los padres y madres y alumnos encuestados afirman tenerlo. Ya no existe un lugar específico donde ubicar los dispositivos tecnológicos. El móvil y las "tabletas" han reestructurado los espacios de uso y se han convertido en protagonistas de la tecnología. Sin embargo, en el contexto del aula la situación se transforma. Los alumnos no pueden utilizar sus dispositivos móviles y no disponen libremente de algún acceso a la red del centro. Resulta significativo que un $80 \%$ de estos afirmen sentirse obligados por el profesorado, según los criterios de evaluación, a usar Internet o el ordenador para realizar los trabajos de sus asignaturas cuando en los mismos cuestionarios el $42 \%$ del profesorado afirma no hacerlo nunca y, un $36 \%$, en ocasiones. Por otro lado, las familias tienen una ligera idea del uso que se hace de las TIC en el Colegio y solo saben lo que les cuentan sus hijos. Sin embargo, no es una queja; tampoco tienen mayor interés. Les preocupa, fundamentalmente, el rendimiento académico que se vea reflejado en las calificaciones. Aunque les inquietan los peligros generados por la participación en redes sociales, los padres y madres comprenden que forma parte de la construcción social de su propia identidad, aunque les gustaría que fuese de otro modo. Sin embargo, en la práctica, el control parental es escaso o nulo y se sienten impotentes para ejercerlo con garantías, delegando dicha responsabilidad al centro académico, como así ha ocurrido con los iPads en Bachillerato. En este sentido, el $98,1 \%$ de padres y madres creen que es necesario que el profesorado eduque al alumnado para un uso correcto de las redes sociales. El cualquier caso, el indicador de seguridad ha sido relegado exclusivamente al rendimiento académico convirtiéndose este en moneda de cambio mediante procesos de duras negociaciones familiares. Este rol ha sido asumido por ambas partes de los agentes familiares; en el Colegio, la relación con el móvil es más compleja y se generan muchas más resistencias, aún siendo este el enclave estratégico. El 47,4\% del profesorado utiliza herramientas tecnológicas a diario y sólo un $20 \%$ hace apenas uso de ellas. Es curioso que tan solo un 18,4\% reconozca participar de algún proyecto relacionado con TIC aún a pesar de que un $47,4 \%$ se considera bien formado. En cualquier caso, la opinión que se tiene sobre las TIC es positiva. Y, aunque 
creen que el Estado debería asumir la responsabilidad formativa del profesorado (76,3\%), consideran imprescindible que este último cambie su actitud ante el uso de las TIC en el contexto educativo $(71,1 \%)$, pues consideran $(63,2 \%)$ que las TIC son el futuro de la educación, como así también lo creen los padres y madres. El alumnado está más inmerso en las TIC pero no ve tan imprescindible su uso en el ámbito escolar, resistencia inducida por los mayores. Los padres y madres creen que si potencian el aprendizaje, mejor; los alumnos/as confían más en sus medios que en la tecnología (por no saber gestionarla); y el profesorado es escéptico ante la responsabilidad que supone su inclusión. Todos piensan lo mismo, todos la utilizan igual pero se expresa de diferente forma.

\section{2: Contradicciones $y$ desencuentros}

Sin embargo, no todos los agentes hacen lo mismo. Hay una coincidencia exagerada en las perspectivas de futuro que otorga la tecnología, influenciada, básicamente, por la inercia socio-cultural. Los padres y madres, que vertían las más duras críticas sobre el uso del móvil, son los que se encuentran menos distanciados tecnológicamente. Léase como nos los afirma una madre cuando le preguntábamos por el uso que hace del teléfono móvil:

Pero tiene su ventaja. Yo tengo aquí que mi prima Paqui me lo acaba de enviar. Si no fuese por esto, mi prima Paqui que vive en Guadix, pues desde hace dos años y medio que murió mi padre, no tendríamos contacto. $Y$ sin embargo, todos los días hablamos, todos los días, todos los días. Ahora mismo no tener WhatsApp es decir cómo me pongo en contacto con ella. Es más simpático, mucho más que un SMS. No interrumpes nada, lo envías y te contesta cuando pueda (Madre de Everest, alumno del centro).

Su inclusión en lo tecnológico es lenta, aunque segura. El hándicap actual radica en la velocidad de las transformaciones socio tecnológicas. Las familias han integrado plenamente el uso del móvil en el hogar familiar y se ha convertido en un ritual cuya inclusión es inevitable. Ha pasado, además, a ser una necesidad familiar, pues en ellas su uso se ha vuelto completamente imprescindible y se ha convertido en una herramienta plenamente horizontal reguladora de las relaciones familiares, como se ha visto en el pensamiento de las familias, relegando las tensiones al ámbito académico:

De hecho hay veces estoy yo con rosa en la casa y la llamo, y digo, voy a tener que escribir un WhatsApp porque nosotros tenemos un pasillo largo y por no darle un... digo que le mando un WhatsApp y seguro que me contesta que si le llamo por su nombre (Madre de Dora, alumna del centro).

El profesorado, en este sentido, se debate entre el cumplimiento de las normas institucionales y la inercia cultural, entre el ROF (Reglamento de Orden y Funcionamiento del Centro) y la necesidad. Unos ganan terreno y otros lo pierden, acercando distancias, aunando intereses. El 47,4\% del profesorado no confía en que en el futuro se trabaje en exclusiva con Internet y dispositivos móviles, el mismo porcentaje que considera que el teléfono móvil podría contribuir en algo a la organización académica de su centro de trabajo. La opinión del profesorado está dividida. Un 21,1\% de los profesores dicen haber dejado utilizar alguna vez el teléfono móvil a sus alumnos en clase, aunque la realidad es que entre pasillos, la opinión generalizada era de persecución y fiscalización. Esta visión contrasta con la consideración (un 76,3\%) de que los profesores deberían educar para un uso correcto, y lleva a pensar en una evidente falta de confianza en el uso que estos hacen. Las dos mayores restricciones que el centro tiene hecho a los iPads en Bachillerato son la cámara iSight y la 
descarga de aplicaciones, la conexión a las redes sociales y a ciertos contenidos de Internet, con lo que cual resulta significativo, pues en el fondo, el problema es de formación y miedo a la pérdida del control sobre el conocimiento y no tecnofóbico.

\subsection{El refuerzo de tópicos y actitudes}

El discurso del alumnado está muy influenciado por el de los adultos. La mayoría de los comentarios sobre las ventajas e inconvenientes del iPad y las TIC en el trabajo académico han sido negativos, reforzados por una visión más bien escéptica de estas. Utilizan más que nadie las TIC para todo y sin embargo su pensamiento es excluyente, claramente influenciado por el pensamiento adulto, como demostraban en algunas de las entrevistas:

Bien, pero a mí me gustan estudiar más con libros. Hay gente que le gusta subrayar en papel y se hace resúmenes. No creo que me ayude mucho a trabajar. No sé. Ahí también tienes que concienciarte que el iPad es para trabajar y dejar las tonterías. Porque si no, encima, en bachiller, si no estudias, las notas... El iPad puede ser otra distracción más, pero como lo relacionas que es para el colegio pues no piensas usarlo para otras cosas... No sé... (Pepa, alumna del centro).

Los alumnos expresan estas ideas sobre el iPad, sin embargo son incapaces de prescindir del teléfono móvil pues en ellos se hallan concentrados todos los aspectos de su vida:

Yo: ¿Qué ocurriría si yo os quitara el móvil durante una semana?

N: Ummm, "me cagaría en to lo que se menea" (Suzy, alumna del centro).

Profesorado y padres y madres no contribuyen mucho a su éxito, aún a pesar de que durante las encuestas y las entrevistas los comentarios eran más bien positivos. En los grupos de discusión se refuerza la visión negativa de las TIC y a través de ellos han surgido más temores y dudas, rechazos y reticencias. En los grupos de padres y madres se refuerzan las actitudes de miedo y respeto hacia las TIC.

Ese es el problema del iPad, que nos toca a los padres estar pendientes de otro aparatito más. Y que lo utilicen correctamente (Padre de Rocky, alumno del centro).

Pues de las tecnologías, en realidad, conozco que en bachiller ya se usa el iPad y no sé exactamente si es bueno o malo, porque le dan muy buena publicidad pero... yo todavía no me acabo de creer que estudiar en un iPad sea práctico, no me lo termino de creer. Y..., ¿de otras tecnologías? Pues no sé mucho, no sé mucho y debería usarse más el ordenador, por ejemplo, cuando la Junta regaló los ordenadores no se ha utilizado nada en el Cole. Sé que hay pizarras electrónicas y poco más (Madre de Skye, alumna del centro).

Cuando yo fui a la reunión y había padres muy reacios a la instalación del Ipad. Sobre todo los clásicos: qué va a pasar con el papel, donde se ponga un libro de texto que se quite lo otro... y yo ví que el director se vino atrás con eso (Madre de Emilia, alumna del centro).

En los grupos de alumnos y alumnas se ven reflejados estos miedos debido a la influencia familiar. 
Bien, pero a mí me gustan estudiar más con libros. Hay gente que le gusta subrayar en papel y se hace resúmenes. No creo que me ayude mucho a trabajar. No sé. Ahí también tienes que concienciarte que el iPad es para trabajar y dejar las tonterías. Porque si no, encima, en bachiller, si no estudias, las notas... El iPad puede ser otra distracción más, pero como lo relacionas que es para el colegio pues no piensas usarlo para otras cosas... No sé... (Pepa, alumna del centro).

Algunos profesores son más permeables a las opiniones de los demás, y si varios profesores generan un discurso defensor, automáticamente se ve reforzado por aquellos que suelen reflejar una actitud positiva ante la innovación educativa que no por el uso de los dispositivos tecnológicos.

Al inicio de la implantación de las TIC en el centro, algunos profesores se mostraron reticentes al uso y las quejas se manifestaban sobre la saturación de trabajo y la falta de formación; en ningún momento se generó un discurso sobre la voluntad de aceptar el reto del cambio; tras varios meses de iPad en clase y tras la sucesiva adquisición personal de los iPads por parte del profesorado, prácticamente todo el mundo dispone de este.

Algunos profesores se quejaban bastante, al inicio, por la implantación del iPad como herramienta de trabajo en los cursos de Bachillerato y no recibieron positivamente su inclusión: "Otro invento, este de los iPads..." (Swiper, profesor del centro). Sin embargo, dos años después, el discurso ha cambiado, las quejas se han minimizado y han justificado su uso:

Bueno, mira, ya está, ahí voy con el cacharrito, miro el horario y hago algunas consultas... Pero nada más (Swiper, profesor del centro).

En fin, existe profesorado que utiliza mucho el móvil pero lo prohíbe aún más y otros que son más permisivos aún no siendo grandes adalides de la tecnología.

\section{Contradicciones: entre los usos y las resistencias}

Los datos nos indican que todos los agentes implicados en el proceso de aprendizaje, de un modo directo o indirecto, utilizan, en mayor o menor medida, los dispositivos electrónicos en el ámbito personal y/o profesional. Sin embargo, existen resistencias, aparentemente insalvables, que ralentizan e, incluso, inutilizan su integración en el aula en el que la disciplina, las lógicas de poder por el control de la autoridad y las lógicas horizontales de dichas relaciones que generan, precisamente, conflictos de roles, y que juegan un papel decisivo, tanto en el contexto familiar como en el de aula, inutilizan las posibilidades reales que ofrecen.

Las relaciones de enseñanza-aprendizaje son relaciones de poder, tal y como nosotros las conocemos. La pérdida de control conlleva pérdida de autoridad. La inclusión de las TIC a la realidad de los seres humanos, y en particular, al ámbito educativo, genera conflictos que tienen que ver con estas relaciones de poder y sus lógicas de aplicación. Así es que muchos de los instrumentos y herramientas destinadas a la mejora del aprendizaje, que son sociales y que se pueden aplicar al ámbito escolar, pues sus profesores son los mismos que deambulan en la misma realidad del resto de agentes implicados en el aprendizaje, lo que Margaret Mead llamó culturas prefigurativas, transforman o, al menos, matizan, las relaciones de poder dentro del modelo clásico de enseñanza-aprendizaje, basados en relaciones horizontales frente a la verticalidad de modelos tradicionales, tal y como, intuyó ella que sucedería (Mead, 1971). La lógica cambia. ¿Razones? Sus agentes, no sus contenidos. Muchos de los agentes que dirigen el éxito global de las redes sociales y el uso de la tecnología 
a nivel global se han desintegrado de un sistema estándar que enseña de forma tradicional. Muchos de ellos, como los creadores de WhatsApp o el mismo Steve Jobs (Novoa, 2014), se presentan como ejemplos paradigmáticos del nuevo conocimiento del siglo XXI, conocimiento que trasciende el convencionalismo preinstaurado, prescinde de lo convencional académico, y se autoforma, motivo para la reflexión, nuevos jóvenes desencajados del sistema y que triunfan en una sociedad tecnificada, como lo son un ejemplo de esto, los hackers y los especialistas en videojuegos, que desarrollan nuevas estrategias de aprendizaje en un mundo cuyas habilidades cognitivas del desarrollo se han demostrado a través de los videojuegos. Esto no significa la ausencia de jerarquías, sino la emergencia de nuevas estructuras jerárquicas basadas en modelos de autoridad horizontales y no tan verticales como viene siendo, herencia clara de sistemas claramente verticales como lo son el militar, el sanitario, el administrativo o el académico.

"Encended los móviles" (De Pablos, 2015). Quizá sea esta la frase que debamos oír a partir de ahora en los centros educativos. La innovación educativa, con especial protagonismo de la tecnología, es una realidad. Sin embargo, las resistencias reducen significativamente el impacto de la inclusión de innovación tecnológica en las aulas debido fundamentalmente a:

- Una visión optimista de las nuevas tecnologías aplicadas al ámbito de la educación de parte de los distintos agentes implicados directamente frente a una visión pesimista sobre su uso -sobre la descripción y el reconocimiento que el alumnado, padres y madres y profesorado hacen de los dispositivos móviles en el contexto educativo:

* Alumnado. Percibe continuos mensajes contradictorios sobre las TIC, condicionado por una sociedad hermética basada en modelos de enseñanza-aprendizaje tradicionales y que son los que delimitan la base para los procesos selectivos de cara al acceso a la carrera profesional. Esto hace que existan reticencias. El alto nivel de competitividad exigido por la administración hace que el alumnado no vean en el trabajo cooperativo un beneficio, pues los procesos de selección y competitividad nada tienen que ver con los que se predican en las enseñanzas medias. Sin embargo, este sí percibe grandes beneficios en el uso de las nuevas tecnologías.

* Padres y madres. Creen firmemente, excepto alguna excepción, que las nuevas tecnologías son fundamentales. Sin embargo, lo hacen arrastrados por la inercia tecnológica del mercado, pues la opinión generalizada que hay entre todos los padres y madres es de miedo y respeto hacia el uso de las nuevas tecnologías, con especial atención al teléfono móvil, el dispositivo preferido de los jóvenes. La mayor aportación para domeñar el miedo se encuentra en las consecuencias del éxito o fracaso escolar basado en la ecuación rendimiento-uso.

* Profesorado. Son el colectivo que tiene una situación más delicada en todo este proceso. El profesorado es la pieza clave del cambio. La voluntad por un lado, y la formación, por otro, son los dos pilares sobre los que las transformaciones educativas pueden producirse. La voluntad es la clave de todo el proceso. La actitud de espera a las imposiciones del sistema para ejecutar el cambio es propia de la zona de confort que justifica cualquier acción pasiva. La predisposición de formación y medios a veces debe comprometer al profesional que no puede esperar pasivamente a que el sistema provea de materiales y recursos para la innovación, cuyos procedimientos, a veces, son completamente ajenos 
al sistema de enseñanza pública; basta ver que las grandes plataformas de innovación educativa se gestionan a través de empresas privadas que tienen intereses en el mercado: smconectados, Fundación Telefónica, Fundación Santillana o El País de los Estudiantes; pocas plataformas dependientes de la institución pública tienen tanto impacto como estas. Una vez producida esta situación, en un contexto de contagio, pueden surgir las destrezas innovadoras. Los procesos de formación son imprescindibles. El modo de aprendizaje viene condicionado por los intereses del docente en función de sus habilidades. La potenciación de esto es una tarea del docente que no puede circunscribirse, exclusivamente, al centro educativo. Por otro lado, por ejemplo, de la prueba celebrada por la Thales $^{6}$ a modo de concurso a nivel nacional, aunque suele haber una mayor participación de centros concertados que de públicos, se destaca como factor definitivo para participar de estas jornadas, la predisposición del profesorado, pues coinciden aquellos profesores innovadores que implementan nuevas estrategias de aprendizaje en clase los que animan a su alumnado a participar de dichas jornadas en horas fuera del horario lectivo. Es una prueba más del factor actitud del profesorado para el éxito o fracaso escolar.

- Ciertos síntomas de estrechamiento de la brecha digital -sobre la brecha digital-. La brecha digital se ha estrechado significativamente desde el momento en el que hay padres y madres que reconocen estar más enganchados al WhatsApp (aplicación móvil del corte "chat" que permite comunicación inmediata y transferencia de vídeos e imágenes entre dispositivos móviles) que sus hijos e hijas. En la encuesta sobre el uso de las TIC y las redes sociales, la aplicación que todos los agentes reconocían que más utilizaban era el WhatsApp. Quizá el profesorado, por su rol profesional, utiliza otras al mismo nivel, pero es significativo que el porcentaje de uso de los distintos agentes sociales es el mismo. Todos coinciden en reconocer su facilidad de uso, las posibilidades que ofrecen la ubicuidad y la rapidez de conexión.

- Horizontalidad en las lógicas de poder. Tal y como advirtió Margaret Mead (1971), existe cierta tensión entre las relaciones de autoridad y de poder ante el conflicto de roles generado por la defensa de las distintas representaciones sociales entre los distintos agentes. Por un lado, entre padres y madres e hijos. Por otro, entre profesores y alumnos. Por otro, entre padres y profesores. Todo esto, ante un periodo de máxima horizontalidad que va en aumento. Los padres y madres ante sus hijos, porque se quiere se amigo a padre y compatibilizar dichas representaciones; los padres ante los profesores, que sienten que el Colegio es un lugar de prestación de servicios a clientes con derechos a todo olvidándose de sus principios básicos en la sociedad: educar y formar para la competencia profesional. Por otro lado, la dirección, que intenta mediar entre las partes pero que pretende ofertar un complejo conjunto de servicios para mantener la demanda. Los alumnos ante los profesores, que buscan a un profesor "guay" omnicomprensivo que haga algo más que transmitir contenidos, que sea un

6 Thales es una asociación matemática sin ánimo de lucro que realiza un concurso matemático a nivel nacional. Su organización no está supeditada a ningún tipo de institución pública o privada, sino a todos aquellos profesores que, impartiendo clase en un centro educativo reglado, quiera participar. Su gestión puede estar representada por miembros de la función pública o privada indistintamente. 
agente implicado en su formación pero con tareas fraterlopaternas. Y todo esto, en un clima de horizontalidad y continua comunicación mediante la tecnología que comparten en los mismos espacios y tiempos: padres que llaman a sus hijos por la mañana y se comunican por WhatsApp para preguntarles algo durante el periodo escolar (no todos pero ya lo van haciendo), profesores que están en redes sociales junto a ellos, y alumnos que te preguntan por la mañana si has visto lo que han escrito o puesto durante el fin de semana como si el fin de y la semana fuesen parte de un mismo periodo, sin discriminación ociosa, en una continuidad peligrosa. Alumnos que preguntan y usan las redes sociales para invadir los tiempos personales para preguntar dudas, según le van surgiendo, porque así lo hacen ante sus amigos y sus padres. Y la falta de respuesta genera a veces tensiones y justificaciones innecesarias. Pero esto no solo ocurre en el contexto educativo, está ocurriendo en todos los espacios de la sociedad donde tiene cabida: en la consulta del médico, en la oficina, en bares y restaurantes, en el teatro, etc. En definitiva, conviven la verticalidad de las relaciones sociales en una, cada vez mayor, horizontalidad de los saberes, que creemos, es una realidad que tiene nuestra sociedad ahora mismo: hemos intentado dar reconocimiento a todos los saberes. Esa convivencia entre el conocimiento (horizontalidad) y la estructura (verticalidad) se manifiesta abruptamente y genera tensiones.

- Tópicos generativos negativos automatizados y potenciados en el contexto educativo por la presión social. Se ha tendido a vincular teléfono móvil y redes sociales al mal comportamiento o al fracaso escolar, sin embargo, no es así. Los alumnos de $4^{\circ}$ de Eso que más actividad frecuentaban en los grupos de WhatsApp ofrecían buenos resultados académicos, en algunos de ellos, los que mejor expediente académico tienen. Los padres y madres estaban preocupados por la implementación del iPad en los cursos de bachillerato hasta que la promoción que comenzó la andadura en el curso 2013-2014 se ha examinado de las pruebas de selectividad con un éxito similar a otros años académicos. Los padres y madres han reducido sus temores. El profesorado ha experimentado que los alumnos tengan iPad en clase no significa la pérdida del control o de la autoridad dentro del aula, uno de los mayores temores y críticas cuando la dirección del centro planteó su implementación como principal herramienta educativa.

No se acaba de asumir la realidad de la inercia digital en las aulas. Las resistencias ante el avance tecnológico son un contrasentido incluso para los propios agentes educativos. La parsimonia genera efectos de retroceso que ralentizan la integración en la escuela. La sociedad funciona de formar global pero cuando se traspasa el dintel del colegio todo se transforma, se retroceden años y se paraliza la realidad digital. Las fuerzas de poder, el miedo al cambio, el conflicto de roles, la integración cada vez más activa de los padres y madres en los colegios mediante la tecnología exige un esfuerzo polinuclear de los agentes sociales integrados en el proceso de transformación de la escuela: las familias, por un lado, adaptándose y posibilitando el acceso de los hijos e hijas a las redes y a Internet desde un innovador control parental al que no están acostumbrados, como una carga más de la vida familiar; profesores y profesoras, adaptándose e integrando lo digital en el contexto de aula fiscalizando dispositivos y en retaguardia; el alumnado, comprometiéndose a hacer un uso responsable e integrador de los medios digitales enfocados al aprendizaje mediante duras negociaciones con la familia aunque con escaso éxito, por ahora, y sintiéndose perseguidos por el profesorado ante el hándicap de la necesidad de su uso frente al miedo a la sanción. 
Pero fundamentalmente, la mayor contradicción radica en tener consciencia de las ventajas innegables del avance tecnológico de parte de todos los agentes como algo que forma parte de lo cotidiano y expresa continuas resistencias en el contexto escolar, resistencias que se deben, básicamente, a la innegable convivencia de las lógicas horizontales del conocimiento, al que todo el mundo tiene acceso y que está ganando un mayor reconocimiento y aceptación, y las lógicas verticales de las estructuras estáticas de la sociedad basadas en relaciones de autoridad y control de ese conocimiento.

Es una realidad la evidente inmersión digital como lo es la inmersión lingüística. Volver atrás es como solo querer hablar una lengua. Reconocer la realidad digital no es difícil, excepto si el contexto es el educativo; entonces, las resistencias aparecen como lo hacen ante la innovación educativa. Las razones: confort y miedo a lo desconocido por la perdida del control...

\section{Bibliografía}

Aguado, J. M. (2003). Los nuevos dispositivos tecnológicos de mediación de la experiencia y su repercusión en el relato reflejo del mundo social. Revista Textos de La CiberSociedad, 2. Procedente de http://www.cibersociedad.net

Álvarez de Sotomayor, I. D. (2011). Estilo de vida de los adolescentes en la red social tuenti (p. 139). UNED.

De Pablos, S. P. (2015, February 23). Por qué no hay que apagar el móvil en clase. El País. Procedente de http://www.elpais.com

Fernández Enguita, M. (2013). Aquí no hay química . La difícil relación del profesorado con la tecnología. Panorama Social, 18, 145-157.

Francecs, P. (2015, February 23). La tecnología y la transformación de la escuela. ElPaís.

García García, J. L. (1998). Sobre el significado y las consecuencias de la diversidad cultural. Baeza.

Garrote Pérez de Albéniz, G. (2013). Uso y abuso de tecnologías en adolescentes y su relación con algunas variables de personalidad, estilos de crianza, consumo de alcohol y autopercepción como estudiante. Universidad de Burgos.

Mead, M. (1971). Cultura y compromiso. Estudio sobre la ruptura generacional. Buenos Aires: Granica.

Novoa, J. (2014). La historia de WhatsApp: de la nada absoluta a ser comprados por Facebook. Procedente de http://www.genbeta.com/movil/la-historia-de-whatsapp-de-la-nada-absolutaa-ser-comprados-por-facebook

Orellana, D. M., \& Sánchez, M. C. (2007). Entornos virtuales: nuevos espacios para la investigación cualitativa. Revista Electrónica de La Educación, 8(1).

Ortiz, A. M., Peñaherrera, M., \& Ortega, J. (2012). Percepciones de profesores y estudiantes sobre las TIC. Un estudio de caso. Edutec-E Revista Electrónica de Tecnología Educativa, 41, $1-15$.

Vásquez Rodríguez, F. (2015). La necesidad de orientar el uso didáctico de las nuevas tecnologías. Ruta Maestra, 12.

Velasco, H., \& Díaz de Rada, Á. (2006). La lógica de la investigación etnográfica. Un modelo de trabajo para etnógrafos de la escuela. (p. 301). Madrid: Editorial Trotta.

Velasco Maillo, H. M., García Castaño, F. J., \& Díaz de Rada, Á. (Eds.). (2007). Lecturas de antropología para educadores. El ámbito de la antropología de la educación y de la etnografía escolar. Trotta.

\section{3}

